\title{
Rupture et délamination de films minces
}

Joël Marthelot (jmarthel@mit.edu), Benoît Roman et José Bico

Physique et Mécanique des Milieux Hétérogènes, CNRS UMR 7636, ESPCI, 75231 Paris Cedex 05

Les films minces qui revêtent les surfaces de nos objets quotidiens tendent à rompre selon un motif de type « boue séchée » sous l'effet d'une contraction du film.

Nous décrivons un nouveau mode de rupture d'une couche mince d'épaisseur micrométrique déposée sur un substrat rigide. Ici, lorsque la couche sèche et se craquelle, elle se décolle simultanément, ce qui conduit à des fissures aux motifs étonnamment réguliers comme des spirales ou des allées oscillantes.

Alors que la formation d'un réseau désordonné de fissures est traditionnellement considérée comme néfaste, la grande régularité géométrique des motifs obtenus peut être un outil pour structurer rapidement des surfaces à l'échelle du micron ou du nanomètre.

\section{Fissures oscillantes} \section{dans des couches minces}

Une couche de peinture recouvrant un mur, le revêtement antireflet d'une vitre ou le film conducteur d'une puce électronique sont des couches minces dans développent pendant le processus de dépôt ou de séchage. Le film se rétracte mais reste solidaire du substrat rigide et emmagasine des contraintes de traction. Si ces contraintes sont suffisamment importantes pour excéder la cohésion du film, des fissures se propagent. Elles forment alors un réseau désordonné qui rappelle des craquelures de boue séchée. Mais lorsque nous diminuons l'énergie d'adhésion entre le film et le substrat, nous observons des chemins de fissures étonnamment réguliers qui forment des spirales et des allées de croissants (fig. 1). De telles fissures ont été récemment découvertes sur des miroirs de lesquelles des contraintes mécaniques se

cavités laser ou sur des films conducteurs utilisés dans des dispositifs électroniques flexibles. Curieusement, elles apparaissent en dessous du seuil de contrainte classiquement prédit. D'un point de vue industriel, il est important de pouvoir maintenir l'intégrité de la couche. Nous avons ainsi déterminé les conditions de propagation de ces fissures et étudié leur chemin afin de pouvoir prédire les motifs [1, 2].

\section{Propagation d'une fissure isolée : le critère de Griffith}

La formation de fissures dans des films minces est pilotée par la compétition entre l'énergie élastique contenue dans le film et l'énergie de rupture du matériau. Habituellement, la fissure se propage en ligne droite et relâche les contraintes de traction dans une petite zone autour de la fissure (zone bleu clair dans la figure 3a).
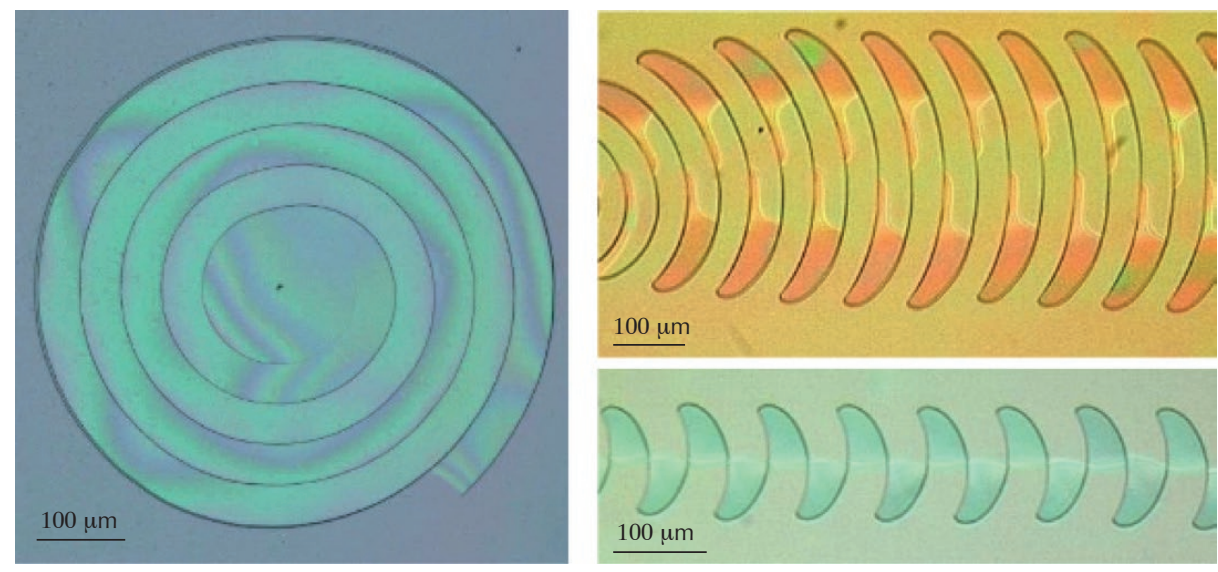

1. Motifs de fissures observés au microscope optique dans des couches minces de silicate d'épaisseur $1 \mu \mathrm{m}$. Les zones de couleur (verte dans la figure de gauche) indiquent un décollement de la couche. La collaboration entre fissuration et délamination permet d'obtenir des chemins de fissures d'une étonnante régularité. 

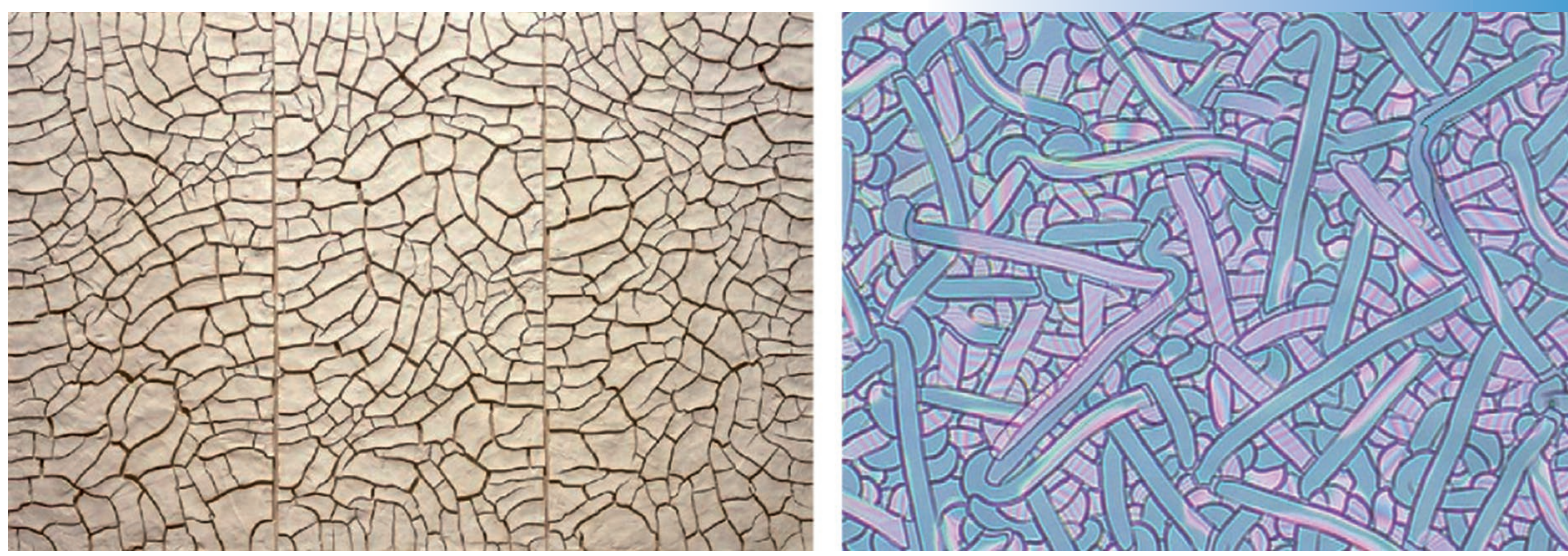

2. Deux exemples de motifs de rupture de couches minces. Gauche, échelle $1 \mathrm{~m}$ : motif de rupture désordonné observé dans une couche de kaolin et de colle vinylique (œuvre d’Alberto Burri, /l viaggio N.4, Fondazione Palazzo Albizzini, Pérouse, Italie). Droite, échelle 1 mm : motif de rupture surprenant que nous observons au microscope optique dans une couche mince de silicate déposée sur un substrat de silicium. Nous remarquons la propagation de bandes d'une largeur bien définie.

Pour prédire les conditions de propagation d'une fissure isolée, nous utilisons un critère énergétique développé en 1921 par Alan Griffith qui s'intéressait à la rupture $\mathrm{du}$ verre. Il propose l'existence d'une énergie de rupture par unité de surface $G_{c}$, analogue à la tension de surface observée dans les liquides. Néanmoins, $G_{c}$ correspond physiquement à une dissipation par unité de surface localisée dans la zone d'endommagement en pointe de fissure et non une énergie de surface thermodynamique comme pour un liquide. Ces dissipations sont, par exemple, dues à l'hétérogénéité de l'interface à petite échelle ou à la plasticité du matériau. Elles sont volumiques; mais comme la région concernée est très petite, nous supposons que sa forme demeure stationnaire lors de la propagation et que $G_{c}$ est une constante caractéristique du matériau. Selon Griffith, la fissure se propage dès que le taux de restitution d'énergie élastique libérée par la propagation compense l'énergie de rupture. Dans le cas d'un film mince d'épaisseur $h$, de module de Young $E$, adhérant à un substrat rigide et soumis à des contraintes $\sigma$, l'énergie élastique libérée lors de la propagation est confinée sur une distance de l'ordre de $h$ autour de la fissure. L'énergie relâchée autour d'une fissure de longueur $L$ est égale au produit de la densité d'énergie élastique $\sigma \times \sigma / E$ dans le volume de film $\gamma h^{2} L$ (représenté en bleu clair dans la figure $3 a$ ), où $\gamma$ est un préfacteur d'ordre unité qui dépend de la différence des propriétés élastiques du film et du substrat. Il y a ainsi propagation si cette énergie est supérieure à l'énergie de fissure $G_{\mathrm{C}} h L: \gamma h^{2} L \sigma^{2} / E>G_{\mathrm{c}} h L$.
La notion de contrainte est cependant tensorielle. Ainsi, seules les contraintes perpendiculaires à la fissure sont libérées. En conséquence, une nouvelle fissure est déviée en s'approchant d'une fissure préexistante et se branche perpendiculairement à l'ancienne. Nous obtenons ainsi les motifs de rupture familiers (fig. 2, gauche) que nous retrouvons sur les vernis, la boue séchée ou les craquelures des peintures [3]. Pour une couche et un substrat donnés, la compétition entre énergie élastique et énergie de rupture sélectionne une épaisseur critique du film, $h_{c}=G_{c} E / \gamma \sigma^{2}$, en dessous de laquelle une fissure isolée ne peut se propager : le film est alors considéré comme stable.

Cette condition est largement acceptée et utilisée par les industriels pour la conception de nouveaux systèmes. Cependant, des fissures inhabituelles laissant apparaître des bandes de largeurs bien définies (fig. 2, droite) se propagent parfois pour des couches plus fines que cette épaisseur critique. Comment expliquer ces craquelures inattendues?

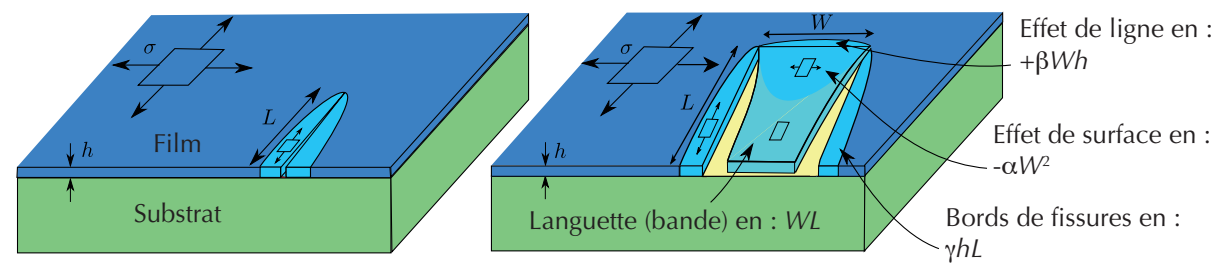

a

b

3. Aire relâchée autour de fissures dans un film mince mis en traction biaxiale $\sigma$ et déposé sur un substrat rigide.

(a) Pour une fissure isolée, l'aire bleu clair où l'énergie est relâchée est confinée dans une petite zone. (b) Pour une bande où fissure et délamination collaborent, l'aire relâchée est beaucoup plus importante. énergétique de Griffith : un duo de fissures

\section{Fissure et délamination collaborent.}

Nous avons étudié le mécanisme de rupture dans une couche de silicate d'épaisseur micrométrique déposée sur un substrat de silicium. De telles couches sont utilisées par exemple pour ajuster les propriétés optiques de cavités laser. Ce produit commercial est optimisé pour éviter la nucléation de fissures. Cependant, quelques points de nucléation sporadiques sont présents sur les échantillons, ce qui nous permet d'étudier des motifs de fissures bien développés et isolés les uns des autres. Au microscope optique, nous observons le phénomène de rupture en temps réel dans des films d'épaisseur inférieure à $h_{c}$ (fig. 4a) . Deux fissures parallèles se propagent de concert, en décollant une bande d'une largeur $W$ bien contrôlée. L'aire libérée dans la bande, représentée en bleu clair dans la figure $3 b$, est beaucoup plus importante que celle relâchée autour de deux fissures isolées. Reprenons l'argument

$\gg>$ 

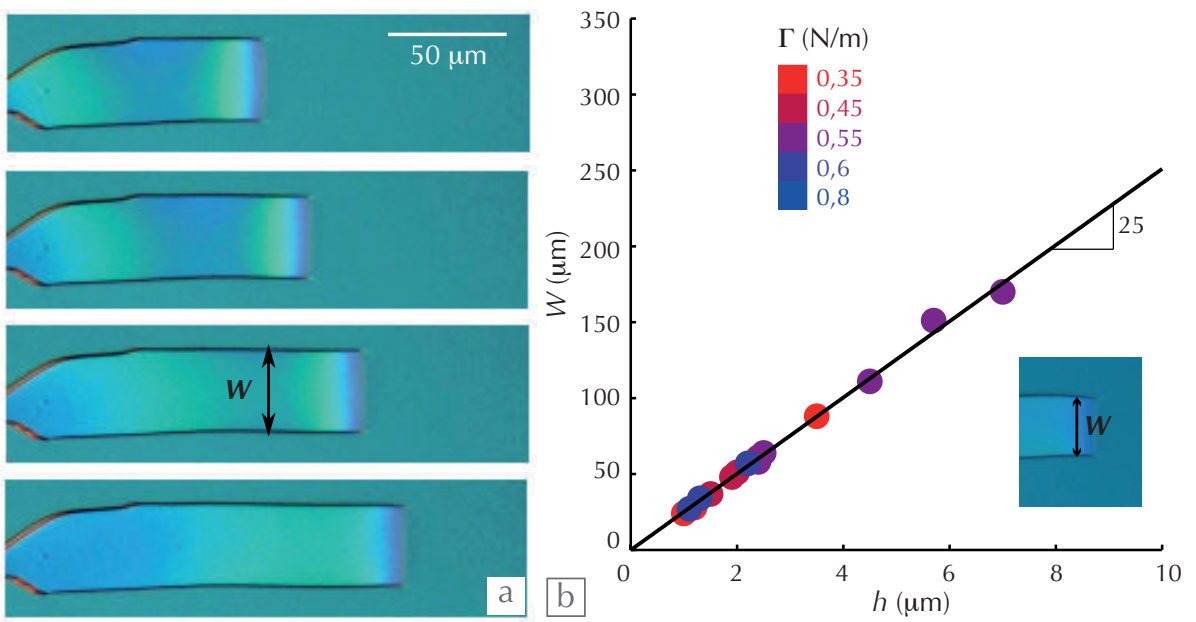

4. Bande formée par un duo de fissures parallèles.

(a) Propagation d'un duo de fissures distantes de $W$.

(b) Largeur $W$ d'une bande formée par un duo de fissures, en fonction de l'épaisseur $h$ du film pour différentes énergies d'adhésion $\Gamma$.

$>>$

peut se propager si le gain d'énergie relâchée dans la bande, $W h L \sigma^{2} / E$, est supérieur à la somme des coûts des deux fissures, $\left(2 G_{c}-2 \gamma h \sigma^{2} / E\right) h L$, et du décollement du film, $\Gamma W L$, où $\Gamma$ correspond à l'énergie d'adhésion du film sur le substrat. La propagation est donc énergétiquement possible si :

$W \geq 2\left(G_{c}-\gamma h \sigma^{2} / E\right) h /\left(h \sigma^{2} / E-\Gamma\right)$.

C'est ainsi la collaboration entre la fissuration et la délamination qui permet à ces bandes de se propager dans des films prédits stables, en libérant davantage d'énergie élastique. Pour une largeur $W$ donnée, le critère de propagation repose donc sur un simple bilan d'énergie, l'énergie élastique libérée compensant la somme de l'énergie de fracture sur les deux côtés de la bande et de l'énergie d'adhésion du film sur le substrat.

La sélection de la largeur de la bande est cependant plus subtile. Nous avons mesuré cette largeur $W$ en régime stationnaire en fonction de l'épaisseur $h$ du film pour différentes valeurs de l'énergie d'adhésion $\Gamma$ (fig. 4b). Curieusement, $W$ augmente linéairement avec l'épaisseur et est indépendante de l'énergie d'adhésion, mais également des propriétés élastiques des matériaux.

Si le critère de Griffith prédit le seuil de propagation, la direction des fissures est dictée par le principe de maximisation du taux de restitution d'énergie. Pour un chargement croissant, la fissure se propage dans la première direction pour laquelle l'énergie élastique libérée atteint l'énergie de rupture du matériau. Dans notre système, deux effets au premier abord insignifiants corrigent l'expression de l'énergie élastique libérée. Ils sont liés au détail des contraintes au voisinage du front de délamination. La bande décollée peut en effet se soulever, ce qui permet de libérer de l'énergie par cisaillement le long du front. Ce petit bonus est proportionnel à la largeur de la bande, $\beta h W$. Cependant, l'extrémité de la bande reste ancrée au substrat. La tension latérale est ainsi restreinte jusqu'à une distance du front de l'ordre de la largeur. Nous obtenons une pénalité proportionnelle au carré de la largeur, $-\alpha W^{2}$. Le gain supplémentaire l'emporte pour les petites largeurs, mais perd pour les grandes.

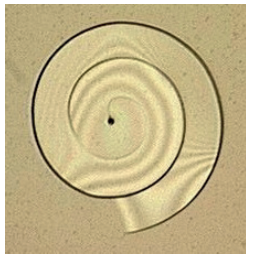

$\mathrm{a}$
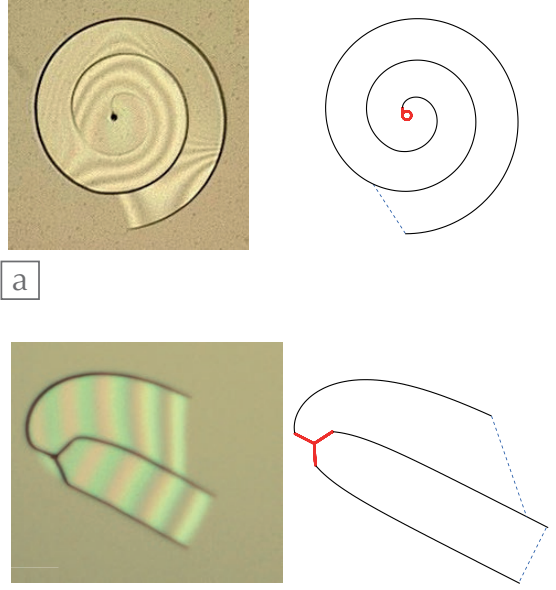
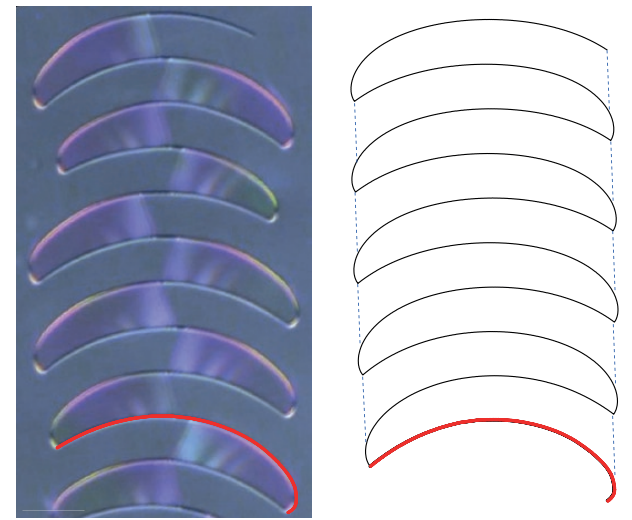

$\mathrm{b}$

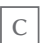

5. Modélisation de chemins de fissures. Notre modèle permet de prédire les chemins de fissures observés à partir de différentes conditions initiales (en rouge) extraites de l'expérience. Une spirale se développe à partir d'un défaut localisé (a), trois fissures à partir d'une amorce à $120^{\circ}$ (b), une allée de fissures oscillantes à partir d'un défaut allongé (c).
L'équilibre des deux effets conduit à une largeur stable, qui est proportionnelle à l'épaisseur du film, $W=(\beta / 2 \alpha) h$. Les préfacteurs $\alpha$ et $\beta$ peuvent être déterminés numériquement par un calcul en éléments finis des déformations élastiques. Alors que le préfacteur $\beta$ est d'ordre unité $(\beta \sim 1,3)$, $\alpha$ est petit $(\alpha \sim 0,025)$, ce qui explique pourquoi la largeur d'équilibre de la bande est significativement plus grande que l'épaisseur du film ( $W \sim 25 h)$, comme nous l'observons expérimentalement (fig. 4b).

Ce mécanisme nous permet de prédire les nouvelles conditions de stabilité du film, que nous avons validées expérimentalement en variant systématiquement l'énergie élastique et les énergies de rupture et d'adhésion du film. Ces critères sont particulièrement importants pour les nouvelles technologies comme l'électronique flexible ou les capteurs biomédicaux, pour lesquels l'adhésion entre couche et substrat est modérée.

\section{Motifs de rupture}

Pourquoi ces motifs en spirale ou en allées de croissants ? Une fissure qui se propage tend à suivre une fissure préexistante à une distance constante. À partir d'un petit défaut arrondi, la fissure se propage ainsi en formant une spirale d'Archimède. Un défaut plus allongé nous conduit en revanche à une allée de croissants. En étendant notre modèle à des géométries plus complexes, nous pouvons prédire de tels chemins de propagation (fig. 5). 

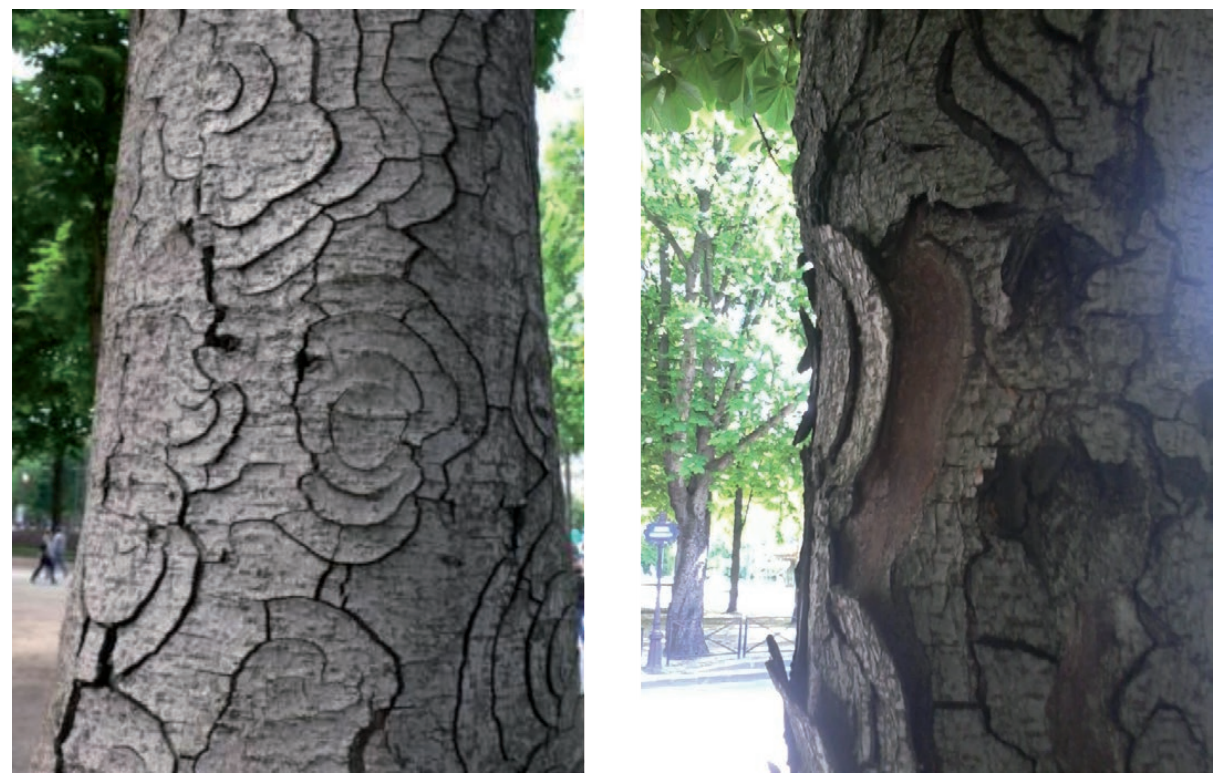

6. Des motifs similaires sont observés dans des écorces d'arbres d'essences courantes (ici, un marronnier rouge du parc de Sceaux, près de Paris).

Nous nous sommes concentrés sur la propagation de fissures dans un matériau dont les conditions de chargement sont bien définies. Cependant, le mécanisme de propagation collaboratif est général et nous l'observons aussi bien dans des films métalliques d'épaisseur nanométrique que dans des couches de vernis ou de peinture épaisses de quelques centaines de micromètres. Curieusement, des motifs similaires marronniers rouges de nos parcs (fig. 6), dans lesquels fracture et délamination semblent collaborer. apparaissent également dans l'écorce des

\section{Les fissures : un outil de fabrication à petite échelle?}

Ce nouveau mécanisme de rupture est un bon candidat pour utiliser les fissures comme un outil de fabrication de structures à petite échelle. Les motifs obtenus se propagent spontanément, sont très robustes, et leur géométrie est dictée par la forme du défaut initial, lorsque les conditions de propagation des fissures sont remplies. Les réseaux de fissures régulièrement espacés peuvent être utilisés pour leurs propriétés optiques, pour fabriquer des canaux microfluidiques ou pour produire des objets (certes délicats!) à l'échelle nanométrique. Le défi majeur qu'il reste à relever est de contrôler la nucléation des motifs. I

\section{Bibliographie \\ 1- J. Marthelot et al., "Self-replicating cracks: a collaborative fracture mode in thin films", Phys. Rev. Lett., 113 (2014) 085502. \\ 2•J. Marthelot et al., "A new failure mechanism in thin film by collaborative fracture and delamination: Interacting duos of cracks", J. Mech. Phys. Solids, 84 (2015) 214-229. \\ 3• L. Pauchard et al., « Craquelures dans les couches picturales des peintures d'art», Reflets de la physique, 3 (2007) 5-9.}

Remerciements

Ces travaux ont été effectués en collaboration avec Jérémie Teisseire et Davy Dalmas dans le laboratoire Surface du Verre et Interfaces du CNRS et Saint-Gobain Recherche, et Francisco Melo à la Universidad de Santiago de Chile. Nous remercions Mélanie Lebental qui nous a initiés à ce mode de propagation, découvert lors de sa thèse au Laboratoire de photonique quantique et moléculaire de l'ENS Cachan. 